\title{
Recent Advances in Cell Micropatterning Techniques for Bioanalytical and Biomedical Sciences
}

\author{
Jun NaKanishi, ${ }^{* 1, * 2 \dagger}$ Tohru TaKarada, ${ }^{* 3}$ Kazuo YamaguchI, ${ }^{* 4}$ and Mizuo MaedA $* 3$ \\ *1 International Center for Young Scientists, National Institute for Materials Science, \\ 1-1 Namiki, Tsukuba, Ibaraki 305-0044, Japan \\ *2 PRESTO, Japan Science and Technology Agency (JST), 4-1-8 Honcho, Kawaguchi, Saitama 332-0012, Japan \\ *3 Bioengineering Laboratory, Discovery Research Institute, RIKEN, \\ 2-1 Hirosawa, Wako, Saitama 351-0198, Japan \\ *4 Department of Materials Science, Faculty of Science, Kanagawa University, \\ 2946 Tsuchiya, Hiratsuka, Kanagawa 259-1293, Japan
}

\begin{abstract}
Cell micropatterning is an important technique for a wide range of applications, such as tissue engineering, cell-based drug screening, and fundamental cell biology studies. This paper overviews cell patterning techniques based on chemically modified substrates with different degrees of cell adhesiveness. In particular, the focus is on dynamic substrates that change their cell adhesiveness in response to external stimuli, such as heat, voltage, and light. Such substrates allow researchers to achieve an in situ alteration of patterns of cell adhesiveness, which is useful for coculturing multiple cell types and analyzing dynamic cellular activities. As an example of dynamic substrates, we introduce a dynamic substrate based on a caged compound, where we accomplished a light-driven alteration of cell adhesiveness and the analysis of a single cell's motility.
\end{abstract}

(Received September 1, 2007; Accepted September 28, 2007; Published January 10, 2008)

\begin{tabular}{llll}
\hline 1 Introduction & 67 & $4 \cdot 1$ Photolithography \\
2 Important Applications & 68 & $4 \cdot 2$ Soft lithography & \\
$2 \cdot 1$ Cellular analyses & & 5 Dynamic Substrates & 69 \\
$2 \cdot 2$ Cell-based sensing & $5 \cdot 1$ Substrates responsive to heat & \\
$2 \cdot 3$ Tissue engineering & $5 \cdot 2$ Substrates responsive to voltage \\
$\begin{array}{l}\text { 3 Materials That Modify Surface Cell Adhesiveness } 68 \\
\text { 3.1 Materials that prevent cell adhesion }\end{array}$ & $5 \cdot 3$ Substrates responsive to light & 71 \\
$3 \cdot 2$ Materials that promote cell adhesion & 6 Caged Culture Substrate & 71 \\
4 Methods to Create Chemical Patterns on Surface & 68 & 7 Conclusions & 71 \\
& & 8 References & \\
\hline
\end{tabular}

\section{Introduction}

Cell micropatterning is a method for controlling the placement of living cells on a substrate surface. ${ }^{1-8}$ It is important for a wide range of applications, such as tissue engineering, cellbased drug screening, and fundamental cell biology studies. Most cell micropatterning methods fall into three categories based on strategy: (1) seeding cells on a chemically patterned surface of different cell adhesiveness, (2) seeding cells on a topographically patterned surface, or (3) directed delivery of cells onto discrete regions of a substrate. Although the latter two categories are also important, this review mainly focuses on

$\dagger$ To whom correspondence should be addressed. E-mail: NAKANISHI.Jun@nims.go.jp the first category. This is because it is the most common strategy and it provides tools to study fundamental aspects of cell adhesion at the single protein/receptor molecule level. ${ }^{9}$ There are excellent reviews that also cover the latter two categories. ${ }^{3,8,10}$ We first introduce some of the important applications of cell micropatterning in general. We then describe methods for micropatterning the cell adhesiveness, referring to materials that promote or inhibit cell adhesion. We then move on to much more sophisticated substrates, so-called "dynamic substrates", whose cell adhesiveness can be changed by an external stimulus, such as heat, voltage and light. As an example of dynamic substrates, we describe a caged culture substrate developed by our group. This type of functional substrate opens up new possibilities in bioanalytical and biomedical sciences. 


\section{Important Applications}

\section{2·1 Cellular analyses}

One of the important aspects of cell micropatterning is its use in understanding basic mechanisms and features of cells and tissues. In the early stage of its history in the 1990's, cell micropatterning was extensively used for neuronal cells in order to construct in vitro models that mimicked the architecture observed in vivo. ${ }^{6}$ In vivo, nerve tissues have cytoarchitecture composed of interconnected neurons and intervening glia cells. However, the network of neurons is lost during the dissociation procedure to form primary cell cultures in vitro. ${ }^{11}$ The circuit of neuronal cells is considered to be of especial importance for higher brain functions, and therefore researchers have tried to reconstitute and investigate the fundamental processes with the aid of bioelectric devices. ${ }^{12-14}$ In the last decade, cell patterning techniques have become much more widely used in the study of endogenous characteristics of non-neuronal cells. This is because cell micropatterning can control not only the location of the cells on a surface, but also the shape, attaching area, and number of contacting cells of each single cell. Those parameters have been found to be quite important in cell survival, ${ }^{15}$ proliferation, ${ }^{16}$ differentiation, ${ }^{17}$ cell migration,,${ }^{18,19}$ cytokinesis, ${ }^{20}$ and cell polarity. ${ }^{21,22}$ In addition, keeping single cells separated by cell micropatterning techniques has been used to analyze variations in gene expression kinetics among individual cells. ${ }^{23}$ On the basis of these achievements, cell micropatterning has currently become one of the most important methods for cell biology research. 5,24

\subsection{Cell-based sensing}

Another important aspect of cell micropatterning is its use in the development of cell-based sensors or cell-based drug testing. ${ }^{4,7,25}$ One example is cell microarrays, which are expected to be new platforms for high-content and highthroughput drug screening in analogy to DNA arrays and protein arrays. ${ }^{25}$ In addition, electrically active cells, such as neurons and cardiac cells, have been used to identify drugs and toxins by detecting their active potentials. ${ }^{26-28}$ For such applications, cell micropatterning is used to increase the proximity of cells to a microelectrode in order to improve the sensitivity. ${ }^{29-31}$ As another application, cell micropatterning was used for the retrieval of B cells reactive to a certain antigen. ${ }^{32}$

\subsection{Tissue engineering}

Tissue engineering is also an important application of cell micropatterning. Cellular activities are highly dependent on their microenvironments, such as their scaffolds and surrounding cells. Therefore, it is important to design and engineer cellular microenvironments so as to make cells in vitro with functional similarities to living tissues and organs. Tissue mimics have been constructed by a cell micropatterning technique, not only for drug screening purposes, but also for future applications for transplantation applications. Hepatocyte spheroids constructed by patterning technology are expected to be an alternative for animal experiments in drug screening. ${ }^{33}$ On the other hand, there is a major technical barrier to the use of micropatterned cells for future transplantation, because of difficulties in dissociating cells from substrates, such as glass, silicon, gold, metal oxides and polymeric materials, without a loss of the constructed structures. Creating cell micropatterns on biocompatible or biodegradable biomaterials ${ }^{34,35}$ or poly $(N$ isopropylacrylamide), ${ }^{36-38}$ a smart material that allows dissociation of a contiguous cell sheet accompanying extracellular matrices, overcomes this issue. In addition to these micropatterned tissue mimics, cultured non-differentiated pluripotent stem cells are an important source for regenerative medicine. From this standpoint, screening of ligand-presenting surfaces that promote the proliferation or selective adhesion of the stem cells on a substrate have also been investigated. ${ }^{39,40}$

\section{Materials That Modify Surface Cell Adhesiveness}

In order to micropattern cells on a chemically modified surface, multiple types of chemical agents with different degrees of cell adhesiveness have to be introduced region-selectively. The present section and the next section first describe materials that modify surface cell adhesiveness, and then ways to introduce those at selected regions of a substrate.

\subsection{Materials that prevent cell adhesion}

Cells such as neurons require specific proteins to attach, and do not grow beyond the proteins. Because of this feature, it is relatively easy to confine them into the regions where such proteins are immobilized. On the other hand, most other cell types show less selectivity against surface chemistry. Even if they show preferential adhesion onto a region at the beginning, the pattern tends to be lost within several hours because nonspecific adsorption of proteins from the media or secreted from cells obscures the surface chemical patterns. Therefore, it is reasonable to use materials that resist protein adsorption to make so-called inert or non-fouling surfaces against the adhesion of cells in general. Bovine serum albumin (BSA), agarose, and phospholipids are natural products that exhibit protein resistance. In addition to such natural products, various synthetic materials have also been developed. ${ }^{41}$ Of these, the most commonly used materials are poly(ethylene glycol) (PEG) and its block copolymer with poly(L-lysine), ${ }^{42}$ poly-lactide, ${ }^{43}$ and polypropylene oxide (Pluronic) ${ }^{44}$ A polymer having phosphocholine, poly(2-methacrylethyl phosphorylcholine) (pMPC), also exhibits good performance. ${ }^{45}$ These materials are physically adsorbed or chemically bound to substrate surfaces. Micropatterning these protein-resisting agents is a key step to micropatterned cell adhesiveness.

\subsection{Materials that promote cell adhesion}

Although it is not always necessary, materials that promote cell adhesion are used in the background of the aforementioned protein-resisting materials. Extracellular matrix proteins such as fibronectin, laminin, and collagen, and polymers having an RGD peptide have cell-adhesive properties. The RGD peptide is a tripeptide of arginine, glycine, and aspartate, which is the major recognition sequence in the extracellular matrix proteins that interact with integrin, a membrane protein..$^{46,47}$ This small peptide sequence is sufficient to promote cell adhesion.

\section{Methods to Create Chemical Patterns on Surface}

To micropattern cell-adhesive and non-cell-adhesive materials described in the previous section, photolithography and soft lithography are commonly used (Fig. 1).

\section{4·1 Photolithography}

Photolithography, developed for fabricating semiconductor devices, is a process for transferring geometric features of a photomask to a substrate by UV irradiation (Fig. 1a). The 
(a) Photolithography

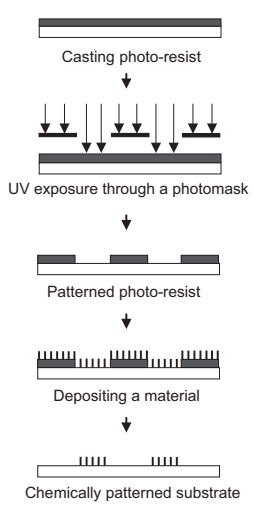

(b) Microcontact printing

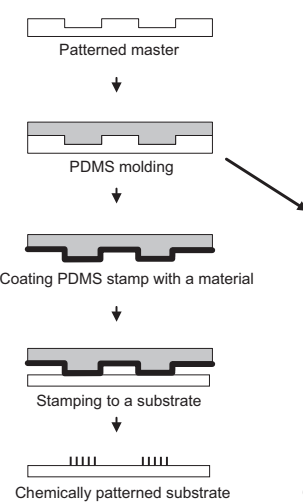

(c) Microfluidic patterning

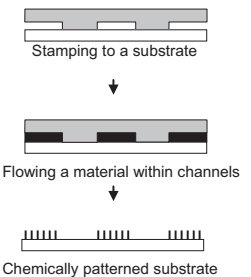

Fig. 1 Schematic illustration of surface micropatterning techniques. Each procedure is discussed in the text.

substrate is coated with a light-sensitive polymer called photoresist and irradiated through a photomask. Depending on its type, the photo-resist becomes either soluble or insoluble by this irradiation. After soluble parts are removed by the developing process, a patterned photo-resist remains. Then, a material of interest is deposited onto the exposed regions of the substrate. Development of the remaining photo-resist yields a micropattern of the material on the substrate.

\subsection{Soft lithography}

Soft lithography is based on a soft elastomeric stamp with patterned relief. ${ }^{48}$ The benefit of soft-lithography is that the photolithographic process is only necessary for the fabrication of a master, and multiple copies of elastomeric stamps can be prepared by replica molding against the master. Because of this, the use of a clean-room environment is minimized; hence, it is more convenient and cheaper than standard photolithography. There are two major soft-lithographic methods for surface patterning: microcontact printing ${ }^{49}$ and microfluidic patterning. ${ }^{50}$

\section{$\mathbf{4 \cdot 2 \cdot 1}$ Microcontact printing}

Microcontact printing is a process of transferring materials from an elastomeric stamp having a patterned relief to a substrate at the area contacted by the stamp (Fig. 1b). By casting a solution of poly(dimethylsiloxane) (PDMS) prepolymer onto a photolithographically fabricated patterned master, a PDMS stamp is replica molded. This stamp is immersed into a solution of the material of interest, and then stamped to a substrate via conformal contact to the surface in order to transfer the material to the substrate. Alkanethiols are often microprinted onto a gold surface to prepare cell micropatterns. ${ }^{51}$ In this case, the microprinted substrates are backfilled with PEG-thiol followed by immersion in a fibronectin solution. This process yields selective adsorption of fibronectin onto alkanethiol-stamped regions, which allows the selective attachment of cells onto the stamped regions.

\subsubsection{Microfluidic patterning}

Microfluidic patterning is a process related to microcontact printing (Fig. 1c). Instead of stamping a PDMS mold having a relief of the master, one having a microfluidic network is stamped to a substrate..$^{50,52}$ By injecting a particular solution through the microchannels, the substrate is exposed to the flow, resulting in patterning of the material. Although this method is less frequently used than microcontact printing, it is more useful to micropattern multiple components on a surface, because

Table 1 Dynamic substrates responsive to external stimuli

\begin{tabular}{cll}
\hline $\begin{array}{c}\text { Type of } \\
\text { external stimulus }\end{array}$ & Stimulus-responsive material & \multicolumn{1}{c}{ Ref. } \\
\hline Heat & PNIPAAm & $34-36,55,56$ \\
Voltage & Hydroquinone & $57-59$, \\
& PEG-thiol & 16,60 \\
& KBr & 61,62 \\
Light & (generates HOBr by oxidation) & \\
& Malachite green & 63 \\
& Spiropyran & 64 \\
& 2-Nitrobenzyl ester & $17,65,66$ \\
\hline
\end{tabular}

single microcontact printing divides the surface into only two regions unless a multilevel stamp is used. ${ }^{53}$ Furthermore, microfluidic patterning allows a directed delivery of cells onto a surface, as well as a selective deposition of materials, as described above. ${ }^{54,55}$

\section{Dynamic Substrates}

Methods described in the previous section produce cellular patterns corresponding to the geometry of masks or masters. In contrast, surfaces whose cell adhesiveness can be changed by an external stimulus, such as heat, voltage, and light, have been studied much more recently (Table 1). Such substrates allow researchers to alter in situ the patterns of cell adhesiveness at any given point in time. This feature is useful for co-culturing multiple cell types and analyzing dynamic cellular activities. For such applications, dynamic substrates should be able to change their cell adhesiveness with no cytotoxic effect on living cells. On the other hand, it is difficult to attain this goal using standard photolithographic methods, because they usually require cytotoxic organic solvents for removing the photoresists.

\subsection{Substrates responsive to heat}

Poly $(N$-isopropylacrylamide) (PNIPAAm) exhibits a temperature-dependent soluble/insoluble change at its lower critical solution temperature (LCST) of $32^{\circ} \mathrm{C}$ in aqueous solution. ${ }^{56}$ Okano and coworkers grafted this polymer onto the surface of a culture dish, and found that it exhibited a hydrophilic/hydrophobic alteration at the LCST, with a corresponding transition from non-cell-adhesive to celladhesive properties. $^{36}$ Cells seeded onto micropatterned PNIPAAm below the LCST only adhered to regions lacking the polymer, and subsequently seeded cells above the LCST filled the remaining surfaces. ${ }^{57}$ This treatment produced regionselective co-culturing of heterotypic cells on the surface. Furthermore, a striking feature of this culture dish is that the cells cultured on this dish can be dissociated as a contiguous cell sheet by decreasing the temperature below the LCST. ${ }^{38}$ This feature is of particular importance in clinical applications. ${ }^{58}$

\subsection{Substrates responsive to voltage}

Mrksich and coworkers have developed dynamic substrates responsive to voltage by introducing an electroactive moiety into self-assembled monolayers (SAMs) formed on a gold surface. They took advantage of the electrochemical oxidation of hydroquinone to benzoquinone, which is reactive to cyclopentadiene by the Diels-Alder reaction. By immobilizing an RGD peptide having a cyclopentadiene group onto a 
background of PEG-thiol after oxidation, and releasing it by reduction, they demonstrated on-off switching of surface cell adhesiveness in both directions..$^{59,60}$ Furthermore, by protecting the hydroquinone by a photocleavable protecting group, they (a)

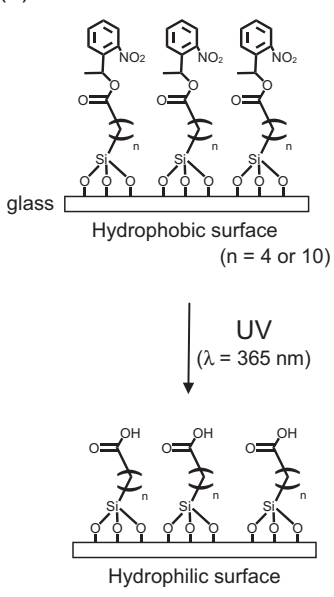

(b)

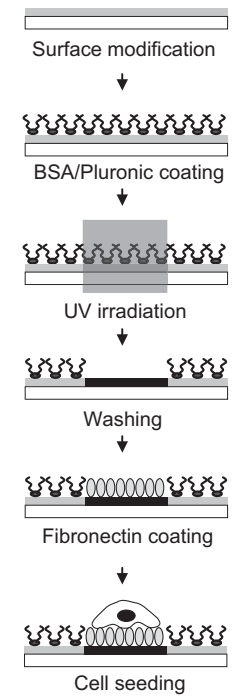

Fig. 2 Caged culture substrate. (a) Photochemical reaction on the substrate surface by UV irradiation at $365 \mathrm{~nm}$. (b) Schematic illustration of cell micropatterning on a caged culture substrate. The procedure is discussed in the text. developed a dynamic substrate in which only the irradiated regions exhibit voltage-dependent cell adhesiveness changes. ${ }^{61}$ On the other hand, Whitesides' group used the electrochemical desorption of PEG-thiol from a gold surface in order to change the surface from non-cell-adhesive to cell-adhesive. ${ }^{62}$ This method has been applied to study the relationship between the direction of cell migration and cell morphology. ${ }^{18}$ Nishizawa and coworkers used the local generation of hypobromous acid $(\mathrm{HOBr})$ from a microelectrode to denature materials that prevent cell adhesion. ${ }^{63,64}$ Like dynamic substrates responsive to light, which are discussed below, this method does not require any preliminary preparation of surface chemical patterns.

\subsection{Substrates responsive to light}

Nakayama et al. have reported a photoactivatable substrate based on malachite green to be a dynamic substrate responsive to light. ${ }^{65}$ Sumaru's group developed spiropyran-grafted PNIPAAm, which showed reversible switching of cell adhesiveness in response to both heat and light. ${ }^{66}$ Compared to heat and voltage, light can be applied to substrates with higher spatial and temporal resolutions. Local regions of the substrates can easily be photoactivated by narrowing the irradiating regions. Therefore, it should be noted that photoactivatable substrates do not require photolithographically or soft lithographically processes to prepare surface chemical patterns. (a)

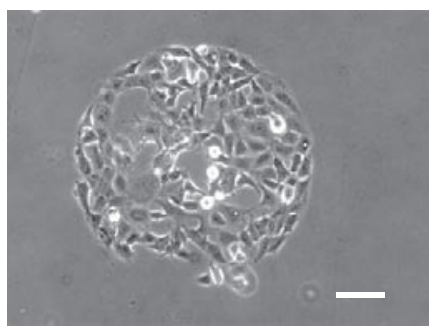

$100 \mu \mathrm{m}$

(b)

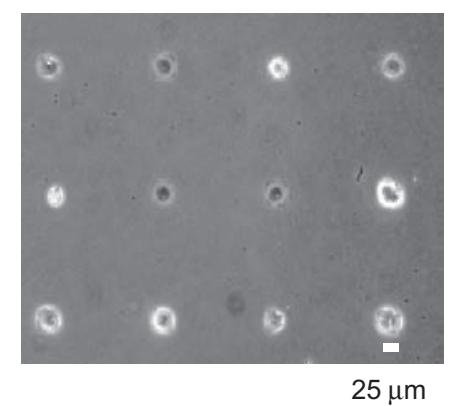

(c)

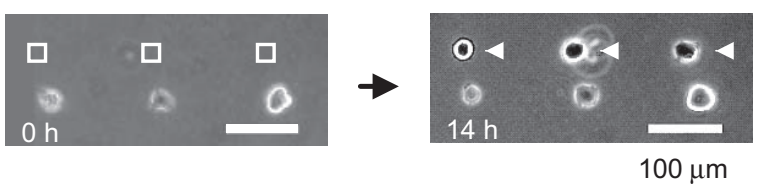

(d) Wide path

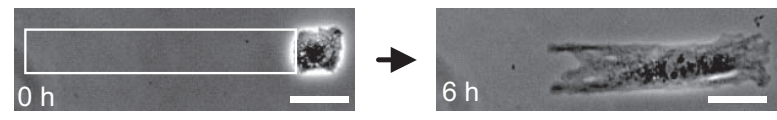

Narrow path

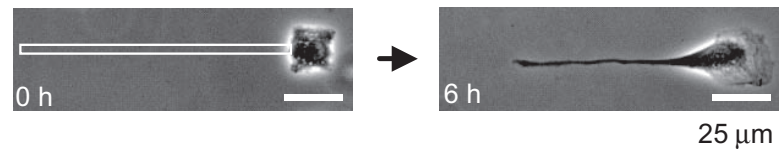

Fig. 3 Dynamic cell micropatterning on a caged culture substrate. (a) Micropatterning of HEK293 cells onto a circular region. A phase-contrast micrograph at $1 \mathrm{~d}$ after cell seeding. (b) Micropatterning of COS7 cells onto a single cell array. A phase-contrast micrograph at $3 \mathrm{~h}$ after cell seeding. (c) Co-culturing of HEK293 cells from different populations at the single cell level. The substrate was irradiated to form a single-cell array of unstained HEK293 cells, and was then again irradiated in proximity to the attached cells, followed by the seeding of fluorescently labeled HEK293. Phase-contrast micrographs before (left) and at 14 $\mathrm{h}$ after second irradiation (right) are shown. Arrowheads represent fluorescently labeled HEK293 cells. White squares in the left image represent irradiated regions. (d) Selective induction of cell migration led by two different protrusions depending on the widths of the irradiated paths. Cells extend lamellipodium-like (upper images) and filopodium-like (lower images) protrusion along wide $(25 \mu \mathrm{m})$ and narrow $(5 \mu \mathrm{m})$ paths, respectively. Phase-contrast micrographs are before (left) and after (right) cell migration induction. White squares in the left images represent irradiated regions. Reproduced with permission from J. Am. Chem. Soc., 2004, 126, 16314, and ibid., 2007, 129, 6694. Copyright 2004 and 2007 American Chemical Society. 


\section{Caged Culture Substrate}

We have developed a dynamic substrate responsive to light, a glass coverslip functionalized with a silane-coupling agent having a caged carboxyl group (Fig. 2a). ${ }^{19,67,68}$ "Caged" means "protected by a photocleavable protecting group". We have termed this dynamic substrate as a caged culture substrate. It was non-cell-adhesive when coated with bovine serum albumin (BSA) or Pluronic, and became cell-adhesive by an addition of fibronectin after irradiation of light $(\lambda=365 \mathrm{~nm})$ (Fig. 2b). The irradiation removed the hydrophobic 2-nitrobenzyl cage from the hydrophilic carboxyl group, leading to the dissociation of BSA or Pluronic that had been adsorbed onto the surface via hydrophobic interactions. Fibronectin was selectively deposited on the irradiated regions, permitting cell attachment corresponding to the irradiated pattern. The photochemical reaction of the 2-nitrobenzyl group by UV light of $365 \mathrm{~nm}$ is widely used in caged compound chemistry to produce physiologically active compounds in living cells. ${ }^{69}$ Their achievements so far guarantee the use of this substrate as a dynamic substrate in high spatiotemporal resolutions with little cytotoxic effect.

We also developed a method to control irradiating regions by inserting a photomask made from a transparency sheet at the field diaphragm of a fluorescence microscope. ${ }^{67}$ In this configuration, the photomask is projected onto the substrate by light from a mercury lamp. The photomask is prepared by printing a pattern on a transparency sheet, and is thus very cheap. By irradiating in this manner, various cellular patterns at single-cell resolution were available on the caged culture substrate (Fig. 3a, b). Subsequent irradiation of the cellpatterned substrate and the seeding of other cells resulted in the co-culturing of single cells (Fig. 3c) ${ }^{67}$ In a similar way, we were able to induce cell migration by irradiating a region alongside the patterned cells. ${ }^{68}$ We applied this method for the selective induction of cell protrusions led by lamellipodia or filopodia along wide and narrow paths, respectively (Fig. 3d). ${ }^{19}$ By tracking the migration of single cells in a microarray format, we performed a quantitative analysis of the cell motility of single cells, and found that cell migration was statistically more rapid in cells led by lamellipodia than in those led by filopodia.

This dynamic substrate is compatible with fluorescence imaging technologies, because the substrate is a glass coverslip suited for observation with an inverted fluorescence microscope and that the photoactivation reaction occurs by the irradiation of light from a mercury lamp. Therefore, it is especially useful as a research tool for cell biology studies to elucidate dynamic biochemical processes during cell migration and cell-cell interactions.

\section{Conclusions}

Cell micropatterning techniques based on chemically modified surfaces were reviewed. Patterned substrates prepared by photolithographic and soft lithographic methods have made great progress in cell biology, cell-based analyses and tissue engineering. In addition to substrates whose patterns are predetermined, dynamic substrates whose cell adhesiveness can be changed by an external stimulus, such as heat, voltage and light have recently been the focus of much more attention. These techniques allow us to gain fundamental biological insights into cellular functions and to use these tissue mimics for pharmacological and therapeutic applications.

\section{References}

1. M. Mrksich and G. M. Whitesides, Annu. Rev. Biophys. Biomol. Struct., 1996, 25, 55.

2. A. Folch and M. Toner, Annu. Rev. Biomed. Eng., 2000, 2, 227.

3. D. R. Jung, R. Kapur, T. Adams, K. A. Giuliano, M. Mrksich, H. G. Craighead, and D. L. Taylor, Crit. Rev. Biotechnol., 2001, 21, 111.

4. K. Bhadriraju and C. S. Chen, Drug Discov. Today, 2002, 7, 612 .

5. M. Mrksich, Curr. Opin. Chem. Biol., 2002, 6, 794.

6. J. M. Corey and E. L. Feldman, Exp. Neurol., 2003, 184, S89.

7. D. Falconnet, G. Csucs, H. M. Grandin, and M. Textor, Biomaterials, 2006, 27, 3044

8. F. L. Yap and Y. Zhang, Biosens. Bioelectron., 2007, 22, 775.

9. M. Arnold, E. A. Cavalcanti-Adam, R. Glass, J. Blummel, W. Eck, M. Kantlehner, H. Kessler, and J. P. Spatz, ChemPhysChem, 2004, 5, 383.

10. H. G. Craighead, C. D. James, and A. M. P. Turner, Curr. Opin. Solid State Mater. Sci., 2001, 5, 177.

11. D. Kleinfeld, K. H. Kahler, and P. E. Hockberger, $J$. Neurosci., 1988, 8, 4098.

12. G. W. Gross, E. Rieske, G. W. Kreutzberg, and A. Meyer, Neurosci. Lett., 1977, 6, 101.

13. S. M. Potter and T. B. DeMarse, J. Neurosci. Meth., 2001, $110,17$.

14. Y. Sugio, K. Kojima, H. Moriguchi, K. Takahashi, T. Kaneko, and K. Yasuda, Sens. Actuators, B, 2004, 99, 156.

15. C. S. Chen, M. Mrksich, S. Huang, G. M. Whitesides, and D. E. Ingber, Science, 1997, 276, 1425.

16. C. M. Nelson and C. S. Chen, FEBS Lett., 2002, 514, 238.

17. R. McBeath, D. M. Pirone, M. N. Celeste, K. Bhadriraju, and C. S. Chen, Dev. Cell, 2004, 6, 483.

18. X. Y. Jiang, D. A. Bruzewicz, A. P. Wong, M. Piel, and G. M. Whitesides, Proc. Natl. Acad. Sci. U. S. A., 2005, 102, 975.

19. J. Nakanishi, Y. Kikuchi, S. Inoue, K. Yamaguchi, T. Takarada, and M. Maeda, J. Am. Chem. Soc., 2007, 129, 6694.

20. M. Thery, V. Racine, A. Pepin, M. Piel, Y. Chen, J. B. Sibarita, and M. Bornens, Nat. Cell Biol., 2005, 7, 947.

21. M. Thery, V. Racine, M. Piel, A. Pepin, A. Dimitrov, Y. Chen, J. B. Sibarita, and M. Bornens, Proc. Natl. Acad. Sci. U. S. A., 2006, 103, 19771.

22. F. C. de Anda, G. Pollarolo, J. S. Da Silva, P. G. Camoletto, F. Feiguin, and C. G. Dotti, Nature, 2005, 436, 704.

23. Y. Kuang, I. Biran, and D. R. Walt, Anal. Chem., 2004, 76, 6282.

24. W. F. Liu and C. S. Chen, Mater. Today, 2005, 8, 28.

25. R. Kapur, K. A. Giuliano, M. Campana, T. Adams, K. Olson, D. Jung, M. Mrksich, C. Vasudevan, and D. L. Taylor, Biomed. Microdev., 1999, 2, 99.

26. G. W. Gross, B. K. Rhoades, H. M. E. Azzazy, and M. C. Wu, Biosens. Bioelectron., 1995, 10, 553.

27. D. A. Stenger, G. W. Gross, E. W. Keefer, K. M. Shaffer, J. D. Andreadis, W. Ma, and J. J. Pancrazio, Trends Biotechnol., 2001, 19, 304.

28. J. J. Pancrazio, J. P. Whelan, D. A. Borkholder, W. Ma, and D. A. Stenger, Ann. Biomed. Eng., 1999, 27, 697

29. J. C. Chang, G. J. Brewer, and B. C. Wheeler, Biosens. 
Bioelectron., 2001, 16, 527.

30. Y. Nam, J. C. Chang, B. C. Wheeler, and G. J. Brewer, IEEE Trans. Biomed. Eng., 2004, 51, 158.

31. C. D. James, A. J. H. Spence, N. M. Dowell-Mesfin, R. J. Hussain, K. L. Smith, H. G. Craighead, M. S. Isaacson, W. Shain, and J. N. Turner, IEEE Trans. Biomed. Eng., 2004, 51,1640 .

32. S. Yamamura, H. Kishi, Y. Tokimitsu, S. Kondo, R. Honda, S. R. Rao, M. Omori, E. Tamiya, and A. Muraguchi, Anal. Chem., 2005, 77, 8050.

33. H. Otsuka, A. Hirano, Y. Nagasaki, T. Okano, Y. Horiike, and K. Kataoka, ChemBioChem, 2004, 5, 850.

34. Y. C. Wang and C. C. Ho, FASEB J., 2004, 18, 525.

35. C. C. Co, Y. C. Wang, and C. C. Ho, J. Am. Chem. Soc., 2005, 127, 1598 .

36. N. Yamada, T. Okano, H. Sakai, F. Karikusa, Y. Sawasaki, and Y. Sakurai, Makromol. Chem. Rapid Commun., 1990, 11,571 .

37. M. A. Nandkumar, M. Yamato, A. Kushida, C. Konno, M. Hirose, A. Kikuchi, and T. Okano, Biomaterials, 2002, 23, 1121.

38. Y. Tsuda, A. Kikuchi, M. Yamato, A. Nakao, Y. Sakurai, M. Umezu, and T. Okano, Biomaterials, 2005, 26, 1885.

39. K. Kato, H. Sato, and H. Iwata, Langmuir, 2005, 21, 7071.

40. B. P. Orner, R. Derda, R. L. Lewis, J. A. Thomson, and L. L. Kiessling, J. Am. Chem. Soc., 2004, 126, 10808.

41. P. Kingshott and H. J. Griesser, Curr. Opin. Solid State Mater. Sci., 1999, 4, 403.

42. S. Pasche, S. M. De Paul, J. Voros, N. D. Spencer, and M. Textor, Langmuir, 2003, 19, 9216.

43. H. Otsuka, Y. Nagasaki, and K. Kataoka, Biomacromolecules, 2000, 1, 39.

44. V. A. Liu, W. E. Jastromb, and S. N. Bhatia, J. Biomed. Mater. Res., 2002, 60, 126.

45. K. Ishihara, N. P. Ziats, B. P. Tierney, N. Nakabayashi, and J. M. Anderson, J. Biomed. Mater. Res., 1991, 25, 1397.

46. E. Ruoslahti, Annu. Rev. Cell Dev. Biol., 1996, 12, 697.

47. S. K. Akiyama and K. M. Yamada, J. Biol. Chem., 1985, $260,402$.

48. Y. N. Xia and G. M. Whitesides, Annu. Rev. Mater. Sci., 1998, 28, 153.

49. A. Kumar and G. M. Whitesides, Appl. Phys. Lett., 1993, 63, 2002.

50. E. Delamarche, A. Bernard, H. Schmid, B. Michel, and H. Biebuyck, Science, 1997, 276, 779.
51. M. Mrksich, L. E. Dike, J. Tien, D. E. Ingber, and G. M. Whitesides, Exp. Cell Res., 1997, 235, 305.

52. E. Delamarche, A. Bernard, H. Schmid, A. Bietsch, B. Michel, and H. Biebuyck, J. Am. Chem. Soc., 1998, 120, 500 .

53. J. Tien, C. M. Nelson, and C. S. Chen, Proc. Natl. Acad. Sci. U. S. A., 2002, 99, 1758.

54. D. T. Chiu, N. L. Jeon, S. Huang, R. S. Kane, C. J. Wargo, I. S. Choi, D. E. Ingber, and G. M. Whitesides, Proc. Natl. Acad. Sci. U. S. A., 2000, 97, 2408.

55. S. Takayama, E. Ostuni, P. LeDuc, K. Naruse, D. E. Ingber, and G. M. Whitesides, Chem. Biol., 2003, 10, 123.

56. M. Heskins and J. E. Guillet, J. Macromol. Sci. Pt. A, 1968, 2, 1441

57. M. Yamato, C. Konno, M. Utsumi, A. Kikuchi, and T. Okano, Biomaterials, 2002, 23, 561.

58. K. Nishida, M. Yamato, Y. Hayashida, K. Watanabe, N. Maeda, H. Watanabe, K. Yamamoto, S. Nagai, A. Kikuchi, Y. Tano, and T. Okano, Transplantation, 2004, 77, 379.

59. M. N. Yousaf, B. T. Houseman, and M. Mrksich, Proc. Natl. Acad. Sci. U. S. A., 2001, 98, 5992.

60. W. S. Yeo, M. N. Yousaf, and M. Mrksich, J. Am. Chem. Soc., 2003, 125, 14994.

61. W. S. Dillmore, M. N. Yousaf, and M. Mrksich, Langmuir, 2004, 20, 7223.

62. X. Y. Jiang, R. Ferrigno, M. Mrksich, and G. M. Whitesides, J. Am. Chem. Soc., 2003, 125, 2366.

63. H. Kaji, M. Kanada, D. Oyamatsu, T. Matsue, and M. Nishizawa, Langmuir, 2004, 20, 16.

64. H. Kaji, T. Kawashima, and M. Nishizawa, Langmuir, 2006, 22, 10784.

65. Y. Nakayama, A. Furumoto, S. Kidoaki, and T. Matsuda, Photochem. Photobiol., 2003, 77, 480.

66. J. Edahiro, K. Sumaru, Y. Tada, K. Ohi, T. Takagi, M. Kameda, T. Shinbo, T. Kanamori, and Y. Yoshimi, Biomacromolecules, 2005, 6, 970.

67. J. Nakanishi, Y. Kikuchi, T. Takarada, H. Nakayama, K. Yamaguchi, and M. Maeda, J. Am. Chem. Soc., 2004, 126, 16314.

68. J. Nakanishi, Y. Kikuchi, T. Takarada, H. Nakayama, K. Yamaguchi, and M. Maeda, Anal. Chim. Acta, 2006, 578, 100.

69. S. R. Adams and R. Y. Tsien, Annu. Rev. Physiol., 1993, 55,755 